\title{
Resistant Streptococcus pneumoniae strains in children with acute otitis media- high risk of persistent colonization after treatment
}

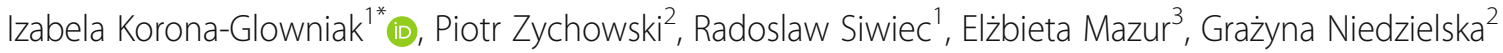 \\ and Anna Malm
}

\begin{abstract}
Background: Despite advances in the development of pneumococcal conjugate vaccines, acute otitis media (AOM) is a common childhood infection, caused mainly by Streptococcus pneumoniae. It has been suggested that persistence of pneumococcal nasopharyngeal carriage is a risk factor for subsequent recurrent infections.

Methods: In this study we evaluate the relationship between 55 pneumococcal strains obtained from nasopharynx/ oropharynx (NP/OP) and middle ear fluid (MEF) of 62 children, aged between 1 and 16 years, during AOM (including recurrent/treatment failure $\mathrm{AOM}$, and post-treatment visits), based on their phenotypic and genotypic characteristics performed by analyses of serotype, antibiotic susceptibility patterns and multilocus sequence typing.

Results: S.pneumoniae was isolated from $27.4 \%$ of MEF samples; it constituted $43.6 \%$ of all positive bacterial samples from MEF samples. There was statistically significant concordance between isolation from the MEF sample and NP/OP colonization by S. pneumoniae $(p<0.0001)$. During post-treatment visits S.pneumoniae was isolated from $20.8 \%$ of children; $91 \%$ of them were positive in pneumococcal NP/OP culture during AOM. The serotypes belonging to 10 - and 13 -valent pneumococcal conjugated vaccines constituted $84 \%$ and $92 \%$ of the strains, respectively. Multidrug resistance was found in $84 \%$ of the strains. According to multivariate analysis, pneumococcal colonization after antibiotic therapy was significantly associated with shorter length of therapy in children with bilateral AOM.
\end{abstract}

Conclusions: High persistent prevalence of antibiotic-resistant S.pneumoniae strains in children with AOM after unsuccessful bacterial eradication may presumably be regarded as a predisposing factor of infection recurrence.

Keywords: Acute otitis media, Streptococcus pneumoniae, Risk factors, Antibiotic resistance, MLST

\section{Background}

Streptococcus pneumoniae is both a commensal bacterium colonizing the human nasopharynx asymptomatically and a pathogen responsible for a wide spectrum of diseases, ranging from mild respiratory tract infections to severe invasive diseases. It is a major cause of morbidity in children and can be isolated in $25-60 \%$ of nasopharyngeal

\footnotetext{
*Correspondence: iza.glowniak@umlub.pl

'Department of Pharmaceutical Microbiology with Laboratory for Microbiological Diagnostics, Medical University of Lublin, Chodzki 1 Street, 20-093 Lublin, Poland

Full list of author information is available at the end of the article
}

samples obtained from healthy children. Nasopharyngeal colonization can lead to infection, by spreading to adjacent mucosal tissue to cause acute otitis media $(\mathrm{AOM})$ or pneumonia, or by blood stream to other sites causing bacteriemia, meningitis or focal infections [1]. The worldwide increase in antibiotic resistance in S. pneumoniae has been related to the spread of several pneumococcal serotypes (6A, 6B, $9 \mathrm{~V}, 14,15 \mathrm{~A}, 19 \mathrm{~F}, 19 \mathrm{~A}$, and 23F), the so-called 'paediatric serotypes' which mainly belong to a small number of pneumococcal clones which nomenclature is standardized by the Pneumococcal Molecular Epidemiology Network (PMEN) [2-5]. 
Considering advances in the development of pneumococcal conjugate vaccines (PCVs) leading to reduction of invasive disorders, pneumococcal diseases are still problem for public health. The impact of the first introduced pneumococcal conjugate vaccine (PCV7) containing seven capsular antigens of serotypes $4,6 \mathrm{~B}, 9 \mathrm{~V}, 14,18 \mathrm{C}, 19 \mathrm{~F}$ and $23 \mathrm{~F}$ on the decrease of invasive pneumococcal disease has been significant in infants, older children and adults. A decrease in rates of antimicrobial resistance among pneumococcal isolates was observed as an additional benefit of the vaccine because resistance to penicillin, macrolides and multidrug resistance are mostly associated with serotypes included in PCV7, namely 6B, $9 \mathrm{~V}, 14,19 \mathrm{~F}$ and 23F [6]. In 2007, World Health Organization (WHO) recommended to introduce PCV to the national infant immunization programs of all countries. Lower proportion of pneumococcal infections caused by PCV7 serotypes has been noted only in countries with routine effective use of PCV7 [7].

Acute otitis media (AOM) is a common childhood infection, occurring most frequently as a consequence of viral upper respiratory tract infections, but treated mainly with antibiotics. The leading causes of bacterial AOM worldwide are Streptococcus pneumoniae, non-typeable Haemophilus influenzae, Moraxella catarrhalis, and group A Streptococcus [8-10]. In approximately $80 \%$ of children aged $2-5$ years AOM is diagnosed at least once, and $30-40 \%$ of them have recurrent episodes $[8,11]$. More than $10 \%$ of children do not improve despite antibiotic therapy. It was noted that history of previous recurrent episodes of AOM, selective pressure of previous antibiotic courses and age below 2 years are risk factors related to this entity [12]. $S$. pneumoniae was shown to be the most prevalent pathogen among AOM patients who had failed a course of antibiotic therapy $[12,13]$. It was demonstrated that the persistence of pneumococcal nasopharyngeal carriage is a risk factor for subsequent recurrent infections and the antimicrobial selection should have an impact on nasopharyngeal colonization $[14,15]$. The nasopharynx is often a reservoir of bacteria involved in AOM where they interact with each other and the host's immune system [16]. Disturbance of existing balance in this microbiota may facilitate the colonizing bacteria to expand through the Eustachian tube into the middle ear and cause infection [17].

In our previous study, we observed the high prevalence of antibiotic-resistant otopathogens in recalcitrant AOM and the high colonization rate by the same otopathogen species after completion of antibiotic therapy [10]. In this study we evaluate the relationship between the pneumococcal strains obtained from the nasopharynx (NP) and oropharynx (OP) and the middle ear fluid (MEF) of children during AOM and during post-treatment visits, based on their phenotypic and genotypic characteristics performed by analyses of serotype, antibiotic susceptibility patterns and multilocus sequence typing (MLST).

\section{Methods \\ Patients}

This prospective study enrolled 62 children, aged between 1 and 16 years who were diagnosed with AOM by an Ear-Nose-and-Throat (ENT) specialist during 2010-2014 in the Department of Pediatric Otolaryngology, Phoniatrics and Audiology, Medical University of Lublin, Poland. From all children's parents, written informed consent was obtained. The Ethical Committee of the Medical University of Lublin approved the study protocol (No. KE-0254/75/ 211). Patients were referred to tympanocentesis according to criteria proposed by Bluestone [18] and extended with recurrent AOM cases. Only a small percentage of patients fulfills the criteria for the tympanocentesis: very severe pain (none in our study), symptoms of toxemia, unsatisfactory reaction to antibiotic therapy (treatment failure), developing complications of AOM, AOM in neonatal and immunosupressed patients (none in our study). All tympanocentesis were performed as an emergency treatment. Patients with recurrent AOM were included only if the microbiological material was taken during active acute inflammatory state, not planned tympanic membrane tube insertion.

A case of AOM was defined when a patient had acute onset of signs lasting 7 or more days with symptoms including fever, otalgia and/or irritability, with presence of middle ear fluid (MEF) and with signs of middle-ear inflammation detected by videootoscopic examination (e.g tympanic membrane erythema and bulging). Recurrent AOM was defined as a history of 3 (or more) episodes in the previous 6 months or 4 (or more) episodes in the previous 12 months; AOM treatment failure defined by persistence of AOM signs and symptoms after at least $48 \mathrm{~h}$ of antibiotic therapy or the recurrence of AOM signs and symptoms within 30 days of completing a course of antibiotics; a new case was defined as the appearance of AOM signs and symptoms after a 30-day symptom-free period after completing a course of antibiotics. A total of $16(25.8 \%)$ children received an ongoing oral antibiotic therapy at the admission to hospital. Children were immunized by a antipneumococcal and/or anti-Haemophilus influenzae type b vaccines in $21 \%$ and $93.5 \%$, respectively. Demographic and selected clinical data of studied children are shown in Table 1. 
Table 1 Demographic and clinical characteristics of children with AOM

\begin{tabular}{|c|c|c|c|c|c|}
\hline \multirow[t]{2}{*}{ Characteristics } & \multirow[t]{2}{*}{ Category } & \multirow{2}{*}{$\begin{array}{l}\text { Total (\%) } \\
n=62\end{array}$} & \multicolumn{3}{|c|}{ No of SP positive samples (\%) } \\
\hline & & & MEF during $A O M$ & NP/OP during $\mathrm{AOM}$ & NP/OP during PT visit ${ }^{c}$ \\
\hline \multirow[t]{3}{*}{ Age (yr) } & $1-2$ & $20(32.3)$ & $6(30.0)$ & $5(25.0)$ & $5(25.0)$ \\
\hline & $3-5$ & $31(50.0)$ & $10(32.3)$ & $11(35.5)$ & $5(21.7)$ \\
\hline & $\geq 6$ & $11(17.7)$ & $1(9.1)$ & $1(9.1)$ & $1(10.0)$ \\
\hline \multirow[t]{2}{*}{ Sex } & Male & $38(61.3)$ & $13(34.2)$ & $12(31.6)$ & $8(24.2)$ \\
\hline & Female & $24(38.7)$ & $4(16.7)$ & $5(20.8)$ & $3(15.0)$ \\
\hline \multirow[t]{3}{*}{ Sibling possessing } & No & $21(33.9)$ & $6(28.6)$ & $5(23.8)$ & $4(23.5)$ \\
\hline & 1 & $30(48.4)$ & $11(36.7)$ & $11(36.7)$ & $7(25.9)$ \\
\hline & $\geq 2$ & $11(17.7)$ & $0(0.0)$ & $1(9.1)$ & $0(0.0)$ \\
\hline DCC/school attendance & & $45(72.6)$ & $12(26.7)$ & $12(26.7)$ & $7(18.9)$ \\
\hline \multirow[t]{2}{*}{ Laterality } & Unilateral & $25(40.3)$ & $5(20.0)$ & $5(20.0)$ & $2(9.1)$ \\
\hline & Bilateral & $37(59.7)$ & $12(32.4)$ & $12(32.4)$ & $9(29.0)$ \\
\hline \multirow[t]{3}{*}{ Category of $\mathrm{AOM}$} & TF & $37(59.7)$ & $10(27.0)$ & $11(29.7)$ & $7(24.1)$ \\
\hline & $\mathrm{R}$ & $6(9.7)$ & $3(50.0)$ & $1(16.7)$ & $1(16.7)$ \\
\hline & N & $19(30.7)$ & $4(21.1)$ & $5(26.3)$ & $3(16.7)$ \\
\hline $\mathrm{CRP}(\mathrm{mg} / \mathrm{L})$ (median, range) $^{\mathrm{a}}$ & & $2.27(0.01-46.9)$ & $3.6(0.15-12.0)$ & $4.8(0.05-19.5)$ & NA \\
\hline WBC (G/L) (median, range) $)^{b}$ & & $14.0(5.4-33.0)$ & $20.4(7.8-30.2)$ & $17.6(7.8-30.2)$ & NA \\
\hline \multirow[t]{2}{*}{ Ongoing antibiotic therapy at the admission to hospital } & No & $46(74.2)$ & $15(32.6)$ & $15(32.6)$ & $10(24.4)$ \\
\hline & Yes & $16(25.8)$ & $2(12.5)$ & $2(12.5)$ & $1(8.3)$ \\
\hline \multirow[t]{4}{*}{ Antibiotic therapy at the hospital } & AM/AMC & $11(17.7)$ & $3(27.3)$ & $1(9.1)$ & $1(11.1)$ \\
\hline & CXM & $21(33.9)$ & $4(19.1)$ & $5(23.8)$ & $4(20.0)$ \\
\hline & CTX/CAZ & $28(45.2)$ & $10(35.7)$ & $11(39.3)$ & $6(26.1)$ \\
\hline & $\mathrm{CLI}$ & $2(3.2)$ & $0(0.0)$ & $0(0.0)$ & $0(0.0)$ \\
\hline Antipneumococcal vaccination & & $13(21.0)$ & $1(7.7)$ & $0(0.0)$ & $0(0.0)$ \\
\hline AntiHib vaccination & & $58(93.5)$ & $17(29.3)$ & $17(29.3)$ & $11(22.5)$ \\
\hline
\end{tabular}

${ }^{a}$ data available for 50 persons; ${ }^{b}$ data available for 61 persons; ${ }^{c}$ data available for 53 persons; $R$ recurrent AOM, TF treatment failure AOM, $N$ new case AOM, MEF middle ear fluid, DCC day care center, CRP C-reactive protein, WBC white blood cells, PT visit post-treatment visit, Hib Haemophilus influenzae type b, $N P$ nasopharyngeal, $O P$ oropharyngeal, $A M / A M C$ amoxicillin or amoxicillin-clavulanic acid, $C X M$ cefuroxime, CTX/CAZ cefotaxime or ceftazidime, $C L I$ clindamicin, NA not applicable

\section{Study procedures}

NP and OP swabs, and MEF specimens were collected at the same time during AOM from 62 children. Next, first-line or second-line antibiotic treatment was administered for the average period of 9 days (median 9, range 4-23, mean 9.6, Standard Deviation (SD) \pm 3.2 ) with the use of amoxicillin or amoxicillin-clavulanic acid, cefuroxime, cefotaxime or ceftazidime and clindamicin (Table 1). Two post-treatment visits were carried out: after the antibiotic therapy (post-treatment visit I) and two weeks after the first visit (post-treatment visit II). All post-treatment visits were performed by ENT specialist (the same for all patients). Medical interview, videotoscopic evaluation of tympanic membrane, tympanometry and hearing level examinations (otoacoustic emisions, pure tone audiometry - depending on patients age and cooperation) were provided during these visits as well as collection of both NP and OP swabs. 28 patients participated in both visits and 53 patients only in post-treatment visit I. A total of 382 microbiological cultures were analyzed (Fig. 1). Due to small number of patients in the post-treatment visit II only the data from post-treatment visit I were included to further statistical analysis.

The nasopharyngeal (NP) and oropharyngeal (OP) specimens were obtained with the sterile alginate-tipped swabs on aluminium or plastic shafts, respectively. Antisepsis of the ear canal before tympanocentesis was performed by installing drain soaked with Octenisept (Schülke \& Mayr) for $1 \mathrm{~min}$. After removing it by suction, myringotomy was carried out, during which dense, mucopurulent discharge draining under pressure from middle ear was found uni- or bilaterally. MEF samples were collected from the ear with the use of sterile suction needle inserted into the middle ear cavity through the incision in the tympanic membrane. The samples were 


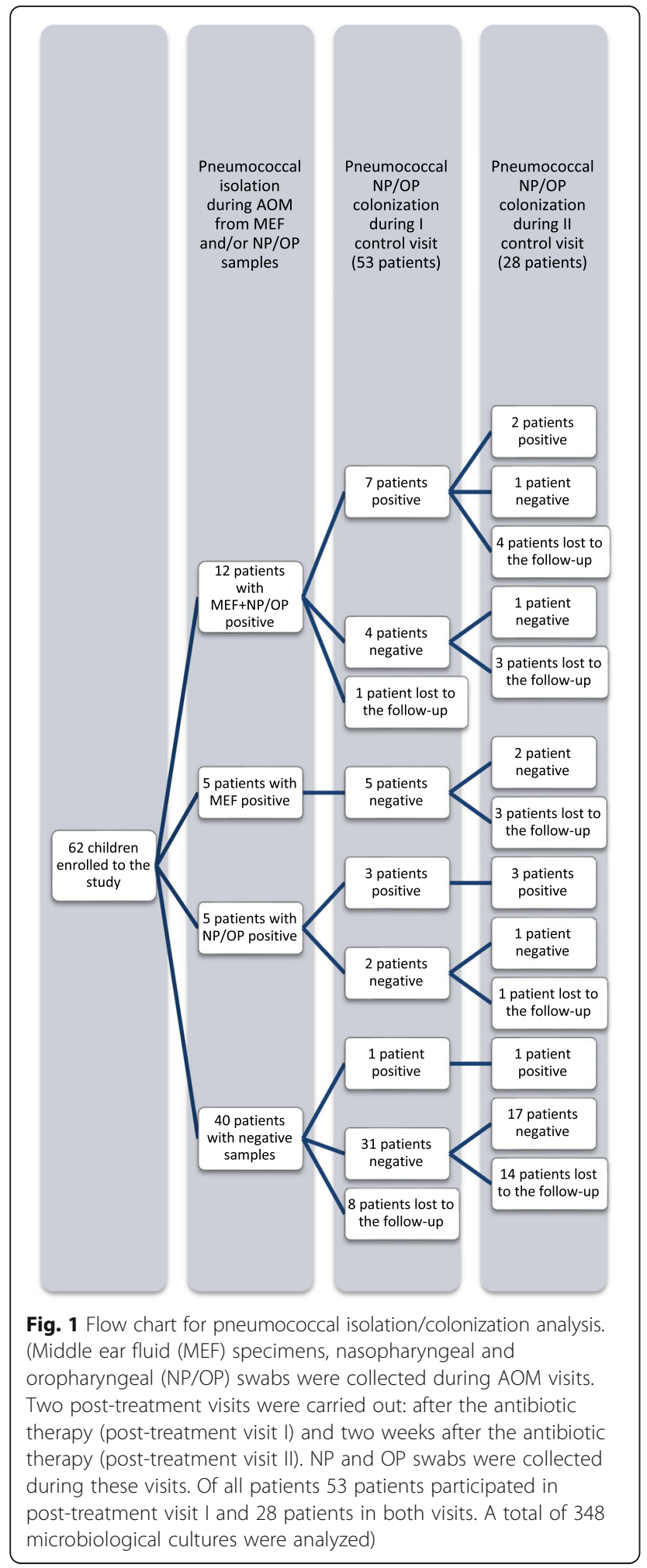

placed in sterile eppendorf tubes maintained in $37^{\circ} \mathrm{C}$, and transported immediately to the laboratory. The remaining amount of exudate had been aspirated. Next, in 29 (46.8\%) patients tympanostomy tube was placed.

\section{Laboratory procedures}

Swabs and MEF samples were inoculated on selective Mueller-Hinton agar with $5 \%$ sheep blood and $5 \mathrm{mg} / \mathrm{L}$ of gentamicin for selective cultivation of streptococci. The identification and serotyping of S. pneumoniae, and susceptibility testing to selected antibiotics were performed in the same manner as in our previous researches $[19,20]$.

\section{Multilocus sequence typing (MLST) analysis}

Bacterial genomic DNA of all 25 isolates was prepared with Genomic Mini Kit (A\&A Biotechnology, Gdynia, Poland) and used as templates for PCR. MLST analyses were performed as described in our previous study [19].

\section{Statistical analysis}

Data processing and analysis were performed using Tibco Statistica Ver. 13.3 (TibcoSoft. Inc.). The results are expressed as percentage or median with range. Univariate analyses were performed using Chi-squared or Fisher exact test, depending on size of samples and of contingency tables for categorical variables and using Mann-Whitney U test for continuous variables. Odds ratios (OR) and their 95\% confidence intervals (CI) were calculated. Logistic regression models were fitted to identify risk factors associated with $S$. pneumoniae AOM cases comparing to other ones. From these models, adjusted odds ratios (OR) and 95\% Confidence Intervals were derived; corresponding $p$-values were those from Wald's test. Goodness of fit was checked using Hosmer and Lemeshow's test. Statistical significance was set if the 2 -tailed $p$ value was $<0.05$.

\section{Results}

The prevalence and affecting factors of $S$. pneumoniae isolations from MEF and NP/OP samples during AOM and post-treatment visits

S. pneumoniae was isolated as a single otopathogen from $22.6 \%$ of MEF cultures and from $4.8 \%$ of MEF samples in polymicrobial cultures. S. pneumoniae was isolated from MEF cultures of 17 (27.4\%) patients and from NP and/or OP (NP/OP) swabs of 17 (27.4\%) patients during AOM. Simultaneous pneumococcal isolation in MEF and NP/OP samples in $12(19.4 \%)$ patients $(70.6 \%$ of $S$. pneumoniae positive MEF samples) revealed statistically significant concordance between isolation from the MEF sample and NP/OP colonization by $S$. pneumoniae during $\mathrm{AOM}(p<0.0001)$.

During the post-treatment visits pneumococcus was isolated from $11(20.8 \%)$ children; 10 of them were positive in pneumococcal culture during AOM. In one patient, S. pneumoniae isolates appeared during the post-treatment visit for the first time (Fig. 1). 
Even though the percentage of pneumococcal isolations was higher from children with treatment failure and recurrent AOM (in MEF sample (31\%) and NP/OP colonization during post-treatment visit $(22.9 \%)$ than from new case AOM (21.1\% and $16.7 \%$, respectively), there was no significant difference in the isolations of $S$. pneumoniae relating to different clinical manifestation of AOM. Frequency of NP/OP colonization by pneumococci during $\mathrm{AOM}$ was nearly equal for patients with treatment failure or recurrent $\mathrm{AOM}$ and new case $\mathrm{AOM}$ (27.9\% and 26.3\%, respectively) (Table 1 ).

According to multivariate analysis, ongoing antibiotic treatment at the admission to hospital was negatively associated risk factor, whereas WBC and CRP values were independent factors positively associated with $S$. pneumoniae as a otopathogen isolated from MEF during AOM (Table 2). Bilateral AOM and shorter length of antibiotic therapy (median 9.0, range 4-12 days, mean $8.2, \mathrm{SD} \pm 2.5$ in colonized patients vs. median 9.5 , range 6-23 days, mean 9.9, $\mathrm{SD} \pm 3.1$ in non-colonized patients) were risk factors associated with pneumococcal colonization after treatment (Table 3).

\section{Pneumococcal strains analysis - Antimicrobial susceptibility, serotype distribution and vaccines coverage, and molecular typing}

A total of 55 pneumococcal isolates were recovered from MEF (24 isolates, both from one or two ears) and/or $\mathrm{NP} / \mathrm{OP}$ (17 isolates) during AOM and 14 isolates were collected from NP/OP during the post-treatment visits. All of the pneumococcal isolates were identified by phenotyping (serotyping, antimicrobial susceptibility) and genotyping (MLST method) which revealed similarity in most of isolates obtained from different samples from the same patients. Finally, the presence of 25 different pneumococcal strains was confirmed. In terms of isolation time, during $\mathrm{AOM}$ and during post-treatment visits 23 and 12 pneumococcal strains were isolated, respectively.

In all patients but one, in whom more than one isolate of $S$. pneumoniae was obtained both over successive visits or from multiple sites, it was the same pneumococcal strain (Table 4). One patient was colonized by two different strains during I and II post-treatment visits (strains 48A and 48B). Out of 22 patients whose samples were pneumococcal positive during AOM, in 12 (54.5\%) patients the same strain was isolated from MEF and NP/ OP samples during AOM (2-3 isolates from the same patient).

The most frequent among isolated strains were serotypes 14 (10/25 strains; 40\%) and 19F (6/25 strains; $24 \%)$. Serotypes belonging to pneumococcal conjugated vaccines - PCV10 (containing serotypes 1, 4, 5, 6B, 7F, $9 \mathrm{~V}, 14,18 \mathrm{C}, 19 \mathrm{~F}, 23 \mathrm{~F}$ ) and PCV13 (containing serotypes $3,6 \mathrm{~A}, 19 \mathrm{~A}$ additionally to 10 -valent vaccine) constituted $84 \%$ and $92 \%$ of the strains, respectively. Some differences in frequency of serotypes distribution among strains isolated during AOM and during post-treatment visits were observed (Fig. 2).

Susceptibility to all tested antimicrobial agents was observed in $12 \%$ (3/25 strains) of the pneumococcal isolates. These strains belonged to serotypes $3,18 \mathrm{C}, 22 \mathrm{~F}$ ( 1 isolate per each serotype). Among all of the strains, $80 \%$ showed decreased susceptibility to penicillin (PNSSP). Higher level of MIC values was shown for pneumococcal strains isolated during post-treatment visits of the patients after completion of the antibiotic therapy for all tested $\beta$-lactams (Table 5). Three strains had mutated during antibiotic treatment and two of them being intermediate became resistant to penicillin and one became resistant to cefotaxime. Eventually, there were differences during AOM and post-treatment

Table 2 Associations of epidemiological factors to prevalence of Streptococcus pneumoniae in middle ear fluid in children with AOM - univariate and multivariate analysis

\begin{tabular}{|c|c|c|c|c|}
\hline \multirow[t]{2}{*}{ Characteristics } & \multicolumn{2}{|c|}{ Univariate analysis } & \multicolumn{2}{|l|}{ Multivariate model } \\
\hline & $\mathrm{OR}(95 \% \mathrm{Cl})$ & $P$ value & $\mathrm{OR}(95 \% \mathrm{Cl})$ & $P$ value \\
\hline Age (yr) & $0.8(0.61-1.06)$ & 0.13 & & \\
\hline Male sex & $1.6(0.86-3.03)$ & 0.14 & & \\
\hline Sibling possessing & $0.96(0.53-1.72)$ & 0.88 & & \\
\hline DCC/school attendance & $0.93(0.50-1.73)$ & 0.83 & & \\
\hline Bilaterality & $1.38(0.76-2.52)$ & 0.29 & & \\
\hline Category of $\mathrm{AOM}: \mathrm{TF} / \mathrm{R}$ & $1.28(0.67-2.42)$ & 0.46 & $0.05(0.01-0.17)$ & 0.062 \\
\hline CRP (mg/L) & $1.05(0.97-1.14)$ & 0.23 & $1.12(1.01-1.25)$ & $0.039^{*}$ \\
\hline WBC (G/L) & $1.21(1.07-1.36)$ & $0.002^{*}$ & $1.22(1.05-1.42)$ & $0.009^{*}$ \\
\hline Ongoing antibiotic therapy at the admission to hospital & $0.54(0.24-1.21)$ & 0.14 & $0.07(0.006-0.87)$ & $0.038^{*}$ \\
\hline Antipneumococcal vaccination & $0.42(0.14-1.2)$ & 0.10 & $0.059(0.002-1.57)$ & 0.091 \\
\hline
\end{tabular}

*Statistic significance; $R$ recurrent AOM, TF treatment failure AOM, $N$ new case AOM, DCC day care center, CRP C-reactive protein, WBC white blood cells 
Table 3 Associations of epidemiological factors to Streptococcus pneumoniae colonization in children with AOM after treatment univariate and multivariate analysis

\begin{tabular}{|c|c|c|c|c|}
\hline \multirow[t]{2}{*}{ Characteristics } & \multicolumn{2}{|l|}{ Univariate analysis } & \multicolumn{2}{|c|}{ Multivariate model } \\
\hline & $\mathrm{OR}(95 \% \mathrm{Cl})$ & $P$ value & OR $(95 \% \mathrm{Cl})$ & $P$ value \\
\hline Age $(y r)$ & $0.8(0.57-1.12)$ & 0.20 & & \\
\hline Male sex & $1.35(0.65-2.8)$ & 0.43 & & \\
\hline Sibling possessing & $0.89(0.44-1.78)$ & 0.73 & & \\
\hline DCC/school attendance & $0.84(0.42-1.68)$ & 0.62 & & \\
\hline Bilaterality & $2.02(0.9-4.6)$ & 0.094 & $3.8(1.1-13.0)$ & $0.037^{*}$ \\
\hline Category of AOM:TF/R & $1.21(0.58-2.54)$ & 0.60 & & \\
\hline CRP (mg/L) & $0.95(0.82-1.09)$ & 0.43 & & \\
\hline WBC (G/L) & $0.98(0.87-1.1)$ & 0.72 & & \\
\hline Ongoing antibiotic therapy at the admission to hospital & $0.53(0.18-1.57)$ & 0.25 & & \\
\hline Antibiotic therapy at the hospital & $1.4(0.57-3.46)$ & 0.46 & & \\
\hline Length of antibiotic therapy at the hospital & $0.73(0.51-1.04)$ & 0.08 & $0.8(0.71-0.93)$ & $0.003^{*}$ \\
\hline
\end{tabular}

*Statistic significance; $R$ recurrent AOM, TF treatment failure AOM, $N$ new case AOM, DCC day care center, CRP C-reactive protein, WBC white blood cells

visits in prevalence of intermediate and resistant pneumococcal isolates to penicillin $(65.3 \%$ and $13 \%$ vs. $66.7 \%$ and $33.3 \%$ ), to ampicillin $(30.4 \%$ and $26.1 \%$ vs. $41.7 \%$ and $33.3 \%)$, to cefaclor $(21.7 \%$ and $78.3 \%$ vs. $0 \%$ and $100 \%)$, and to cefotaxime $(39.1 \%$ and $0 \%$ vs. $50 \%$ and $8.3 \%$ ), respectively. $84 \%$ of $S$. pneumoniae strains were multidrug resistant (MDR-SP). Most of MDR-SP strains $(90.5 \%)$ were non-susceptible to penicillin. Resistance analysis has revealed higher percentage of resistant strains isolated during post-treatment visits in comparison to strains isolated during AOM (Fig. 3). It was shown that among PNSSP, erythromycin- and clindamicin-resistant strains in $90 \%$ belonged to PCV10 serotypes and in $95 \%$ to PCV13 serotypes. When it comes to strains resistant to tetracycline, chloramphenicol and co-trimoxazole, coverage by PCV serotypes was from 86 to $100 \%$.

Among 17 sequence types (STs) identified, 2 STs were newly assigned - ST10340 and ST10342 (Table 4). The eBURST analysis by comparing tested strain collection MLST data with whole MLST database revealed that all of the STs were grouped into 10 clonal complexes (CCs). Four of the CCs containing 7 STs with 14 (56\%) isolates belonged to the pandemic PMEN clones or their variants (SLV and DLV), included Spain9V-3-ST156 (6 isolates), England14-9-ST9 (5 isolates), Spain23F-1-ST81 (2 isolates) and Sweden15A-25-ST63-SVL (Additional file 1).

\section{Discussion}

In this study of pneumococcal aetiology in children with different cases of AOM, high frequency of strains resistant to various antibiotic drugs was found. In fact, among all, $73.3 \%$ of isolated pneumococcal strains were obtained from patients with treatment failure and recurrent AOM. Antibiotics are prescribed at up to $80 \%$ of
GP or ENT visits for AOM whereas antibiotic treatment is considered to be a selection factor of initially non-susceptible strains, facilitating colonization with new strains or development of resistance during therapy. S. pneumoniae was most prevalent pathogen isolated from MEF in patients who had failed a course of antibiotic therapy [13, 21]. Pichichero et al. [22] reported that $28 \%$ of AOM cases were caused by S. pneumoniae with high proportion of highly resistant strains and in $77 \%$ of cases isolation was done from recurrent AOM or initial treatment failure. Eldan et al. [23] found a significantly higher carriage rate of drug-resistant S. pneumoniae in patients with nonresponsive AOM than in children with simple, uncomplicated AOM who did not receive previous antibiotic therapy. In our study, 91.7\% strains isolated from nasopharynx of patients with treatment failure and recurrent AOM were MDR ones. Complete concordance between resistance patterns and STs of pneumococcal isolates recovered from NP/OP and those isolated from MEF has proved that nasopharyngeal MDR strains were the etiologic agents of AOM in $70.6 \%(12 / 17)$ of patients with pneumococcal aetiology. Moreover, all of the pneumococcal isolates recovered from NP/OP during post-treatment visits after treatment were identical according to resistant patterns and STs with those isolated during AOM from the same patients. It is necessary to use molecular typing methods like MLST to find the genetic relatedness between bacterial isolates originating from middle ear cavity and naso/oropharynx. One study revealed with MLST analysis that $100 \%$ of pair pneumococcal isolates from nasopharyngeal and MEF samples were identical [24]. Using other genetic methods, it was observed that 71.4$80 \%$ of $S$. pneumoniae isolated from paired nasopharynx and MEF showed genetic identity [25, 26]. Our study 
Table 4 Phenotypic and genotypic characteristics of 25 pneumococcal strains isolated from children with AOM

\begin{tabular}{|c|c|c|c|c|c|c|c|c|}
\hline \multirow{3}{*}{$\begin{array}{l}\text { No of } \\
\text { strain }\end{array}$} & \multirow[t]{3}{*}{ Serotype } & \multirow{3}{*}{$\begin{array}{l}\text { Antibiotic resistance } \\
\text { pattern }\end{array}$} & \multicolumn{3}{|c|}{ Site of isolation } & \multirow{3}{*}{$\begin{array}{l}\text { Sequence } \\
\text { type }\end{array}$} & \multirow{3}{*}{$\begin{array}{l}\text { Predicted founder } \\
\text { ST/CC }^{\mathrm{a}}\end{array}$} & \multirow{3}{*}{$\begin{array}{l}\text { Related PMEN } \\
\text { clone }\end{array}$} \\
\hline & & & \multicolumn{2}{|l|}{$\mathrm{AOM}$} & \multirow{2}{*}{$\begin{array}{l}\text { PT visit } \\
\text { NP/OP }\end{array}$} & & & \\
\hline & & & MEF R/L & $\mathrm{NP} / \mathrm{OP}$ & & & & \\
\hline 1 & 14 & PECcSxt & $+/+$ & $+/-$ & $+/-$ & 143 & 156/CC1 & Spain 9 V-3 DLV \\
\hline 2 & $19 F$ & PTeCSxt & $-/+$ & $+/-$ & $-1-$ & 423 & $15 / \mathrm{CC} 3$ & England 14-9 SLV \\
\hline 3 & 14 & PECcTeSxt & $+/-$ & $+/-$ & NA & 156 & $156 /$ CC1 & Spain 9 V-3 \\
\hline 5 & 14 & PECcTeSxt & $+/-$ & $+/-$ & $+/+$ & 156 & 156/CC1 & Spain 9 V-3 \\
\hline 10 & $19 F$ & PECcTeCSxt & $-/+$ & $+/+$ & $-/-$ & 87 & $88 / C C 50$ & \\
\hline 11 & $23 \mathrm{~F}$ & PECcTeCSxt & $+/+$ & $+/-$ & $+/+$ & 2033 & $81 / C C 13$ & Spain 23F-1 SLV \\
\hline 14 & $19 F$ & PECcTeCSxt & $+/-$ & $-1-$ & $-/-$ & 87 & $88 / C C 50$ & \\
\hline 22 & $22 \mathrm{~F}$ & S & $-/-$ & $-/+$ & $-/-$ & 433 & 433/CC23 & \\
\hline 27 & $18 \mathrm{C}$ & S & $-/+$ & $-1-$ & $-/-$ & 496 & 496/CC192 & \\
\hline 28 & $23 \mathrm{~F}$ & PECcTeSxt & $+/+$ & $+/+$ & $+/-$ & 81 & $81 / C C 13$ & Spain 23F-1 \\
\hline 30 & $6 B$ & PECcCSxt & $+/+$ & $-/-$ & $-/-$ & 135 & $473 /$ CC 8 & \\
\hline 31 & 14 & PECcSxt & $+/+$ & $-/-$ & $-/-$ & 143 & 156/CC1 & \\
\hline 33 & $19 \mathrm{~A}$ & PECcTeSxt & $-/-$ & $+/-$ & $-/+$ & 320 & $320 C C 2$ & \\
\hline $35 \mathrm{~A}$ & $19 F$ & PECcSxt & $-/+$ & $-1-$ & $+/-$ & 320 & $320 / C C 2$ & \\
\hline $35 B$ & $19 F$ & PECcSxt & $-/-$ & $+/-$ & $-1-$ & 9062 & $320 / C C 2$ & \\
\hline 41 & 14 & E & $+/-$ & $+/-$ & $-/-$ & 9 & $15 / \mathrm{CC} 3$ & England 14-9 \\
\hline 42 & $15 \mathrm{~A}$ & PECcTe & $-/-$ & $-/+$ & $+/-$ & 374 & $63 / C C 10$ & Sweden 15A-25 SLV \\
\hline 44 & 14 & PECcTe & $-/+$ & $+/-$ & $+/-$ & 10,340 & $156 / \mathrm{CC} 1$ & \\
\hline $48 \mathrm{~A}$ & 14 & PECcSxt & $-1-$ & $-1-$ & $+/-$ & 10,342 & $88 / C C 50$ & \\
\hline $48 \mathrm{~B}$ & 14 & PECcTeCSxt & $-1-$ & $-1-$ & $+/-$ & 15 & $15 / \mathrm{CC} 3$ & England 14-9 SLV \\
\hline 49 & 14 & PECcTeSxt & $-/-$ & $+/-$ & $+/-$ & 156 & 156/CC1 & Spain 9 V-3 \\
\hline 55 & 14 & PECcSxt & $-/+$ & $+/-$ & $+/-$ & 15 & $15 /$ CC3 & England 14-9 SLV \\
\hline 56 & $6 \mathrm{~B}$ & PECcTeCSxt & $-1-$ & $+/-$ & $-1-$ & 135 & $473 / C C 8$ & \\
\hline 60 & 3 & S & $+/+$ & $+/-$ & $-1-$ & 505 & 180/CC16 & \\
\hline 62 & $19 F$ & TeCSxt & $+/+$ & $-/-$ & $-/-$ & 423 & $15 / C C 3$ & England 14-9 SLV \\
\hline
\end{tabular}

MEF middle ear fluid, right (R)/left (L) ear, nasopharyngeal(NP)/oropharyngeal(OP) samples, $P T$ post-treatment, $P$ penicillin; $E$ erythromycin, $C c$ clindamycin, Te tetracycline, C chloramphenicol, Sxt co-trimoxazol, $S$ sensitive to all tested antibiotics, PMEN Pneumococcal Molecular Epidemiology Network, ${ }^{2}$ Predicted founders were assigned by comparing tested strain collection MLST data with whole MLST database

and previous findings emphasize the hypothesis that nasopharynx acts as a reservoir for bacterial pathogens in AOM, including S. pneumoniae.

Tympanocentesis is not routinely performed procedure in Polish children which was the reason of relatively small number of enrolled patients. It is a limitation of this study. However, the results seem to confirm the relationship between the persistent pneumococcal colonization after unsuccessful eradication and presumably recurrence of an infection. It is worth noting that in 5 patients, whose MEF samples were $S$. pneumoniae positive during AOM, pneumococcal eradication was observed during post-treatment visit.

Some studies suggest that signs and symptoms of AOM caused by $S$. pneumoniae may be more severe (fever, severe earache, bulging TM) than those caused by other pathogens [27-29]. As well as it was observed elsewhere [30], in our study, higher values of CRP and $\mathrm{WBC}$, whose levels rise in response to inflammation, were significantly important factors involved presence of S. pneumoniae in MEF sample in children with AOM.

A high level of penicillin-resistant S. pneumoniae is considered today the leading cause of antibiotic therapeutic failures in AOM [13-15, 23]. High frequency of PNSSP isolations in children with recalcitrant AOM, observed in our study, was also reported by other authors in other countries, including Poland [13, 23, 31, 32]. Furthermore, we noticed the difference between frequency of PNSSP isolations during $\mathrm{AOM}$ (86.4\%, 19/22 strains) and during post-treatment visits $(100 \%, 12 / 12$ strains) caused by the selection pressure of used antibiotics. Moreover, in case of three strains isolated during post-treatment visits the increase of penicillin and cefotaxime MIC values were observed in comparison to isolates obtained during AOM. 


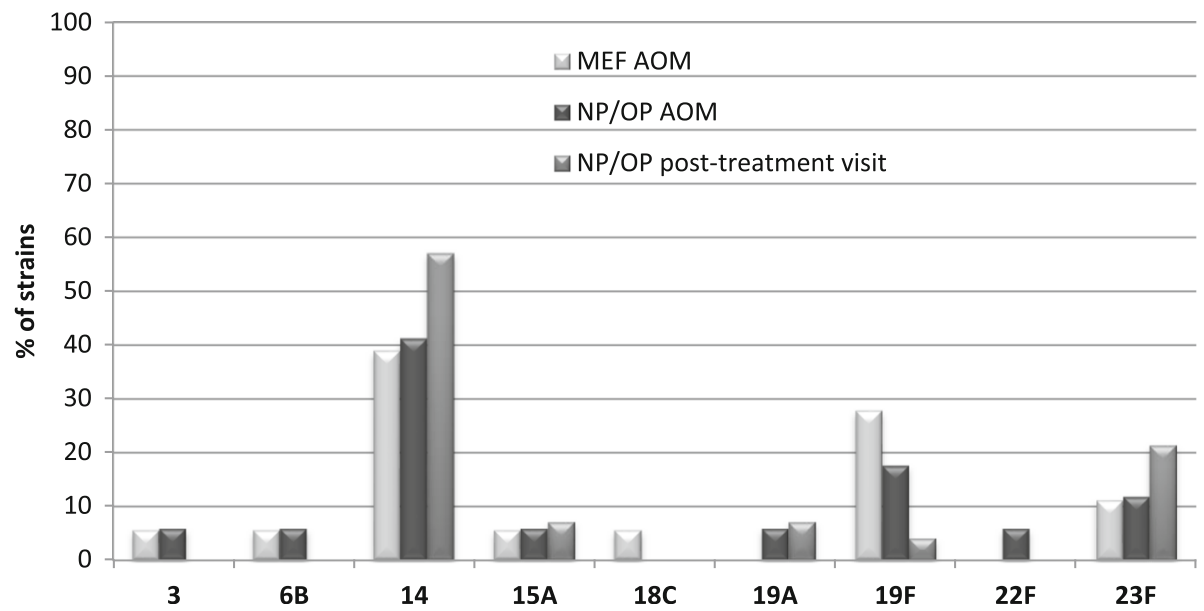

Fig. 2 Serotype distribution for 55 pneumococcal isolates obtained during AOM and post-treatment visits. (AOM, acute otitis media; MEF, middle ear fluid; NP, nasopharyngeal; OP, oropharyngeal)

Very high frequency $(84 \%)$ of MDR strains in our studies has exceeded the data presented in other studies on AOM treatment failure, that $40-67 \%$ of pneumococcal strains were MDR [32, 33]. Our study have confirmed recent reports showing that antibiotic therapy in AOM induces selection of preexisting nasopharyngeal antibiotic-resistant S. pneumoniae [14, 34]. In our study, it was shown that too short time of therapy in children with bilateral AOM, but not of the type of $\beta$-lactams has the impact on persistent pneumococcal colonization after treatment.
In this study, the frequency of $S$ pneumoniae isolation from MEF samples was $27.4 \%$ and it constituted $43.6 \%$ of positive MEF samples. There are similarities between our study and other studies using conventional culture method which reported relatively high rates of pneumococcal MEF isolations from children with recurrent AOM and AOM treatment failure [32, 35]. Presumably, in our study, this situation was directly related to antipneumococcal vaccination pursued only in $21 \%$ of tested children. There was a significantly lower frequency of pneumococcal colonization $(0 \%)$ in immunized children

Table 5 Minimal inhibitory concentrations (MIC) for selected $\beta$-lactam antibiotics for 55 pneumococcal isolates obtained during AOM and post-treatment visits

\begin{tabular}{|c|c|c|c|c|c|c|c|c|c|c|c|c|c|c|c|c|c|c|c|c|c|}
\hline \multirow[t]{2}{*}{ Antibiotics } & & \multicolumn{17}{|c|}{ Number of strains with MIC (mg/L) } & \multirow[t]{2}{*}{ Range } & \multirow[t]{2}{*}{$\mathrm{MIC}_{50}$} & \multirow[t]{2}{*}{$\mathrm{MIC}_{9 \mathrm{c}}$} \\
\hline & & $\leq 0.023$ & 0.064 & 0.12 & 0.19 & 0.38 & 0.5 & 0.75 & 1.0 & 1.5 & 2 & 3 & 416 & $16 \quad 24$ & 32 & 48 & 64 & $\geq 256$ & & & \\
\hline \multirow[t]{3}{*}{ Penicilin } & MEF AOM & 4 & & & 1 & 1 & 2 & 4 & 3 & 1 & 1 & & & & & & & & $0.023-2.0$ & 0.75 & 2.0 \\
\hline & NP/OP AOM & 3 & & 1 & 1 & & 2 & 4 & 3 & 1 & 2 & & & & & & & & $0.023-2.0$ & 0.75 & 2.0 \\
\hline & PT visit & & & & 1 & 2 & 1 & & 4 & & 3 & 1 & & & & & & & $0.19-2.0$ & 1.0 & 2.0 \\
\hline \multirow[t]{3}{*}{ Ampicillin } & MEF AOM & 4 & & & 1 & 2 & & 2 & & 5 & 1 & 2 & & & & & & & $0.023-3.0$ & 0.75 & 3.0 \\
\hline & NP/OP AOM & 3 & & 1 & 1 & 2 & & 2 & & 4 & 2 & 2 & & & & & & & $0.023-3.0$ & 0.75 & 3.0 \\
\hline & PT visit & & & 1 & & 2 & & 2 & 1 & 2 & 1 & 2 & 1 & & & & & & $0.12-4.0$ & 1.0 & 3.0 \\
\hline \multirow[t]{3}{*}{ Cefaclor } & MEF AOM & & & & & 4 & & 1 & & & 1 & & & 2 & 5 & 1 & 1 & 1 & $0.38-256$ & 24 & 64 \\
\hline & NP/OP AOM & & & & & 3 & 1 & & & 1 & 1 & & & 2 & 5 & 1 & & 2 & $0.38-256$ & 24 & 256 \\
\hline & PT visit & & & & & & & & & 1 & & 1 & & 2 & 3 & 1 & 1 & 2 & $1.5-256$ & 32 & 256 \\
\hline \multirow[t]{3}{*}{ Cefotaxime } & MEF AOM & 4 & & 1 & 2 & 1 & 3 & 4 & 1 & 1 & & & & & & & & & $0.023-1.5$ & 0.5 & 1.0 \\
\hline & NP/OP AOM & 3 & 1 & 1 & 2 & 1 & 3 & 3 & 1 & 1 & 1 & & & & & & & & $0.023-2.0$ & 0.5 & 1.5 \\
\hline & PT visit & & & 1 & 1 & 1 & 2 & 4 & 2 & & 1 & & & & & & & & $0.12-2.0$ & 0.75 & 1.0 \\
\hline \multirow[t]{3}{*}{ Imipenem } & MEF AOM & 4 & 2 & 3 & 3 & 5 & & & & & & & & & & & & & $0.023-0.38$ & 0.12 & 0.38 \\
\hline & NP/OP AOM & 3 & 3 & 3 & 2 & 5 & & 1 & & & & & & & & & & & $0.023-0.75$ & 0.12 & 0.38 \\
\hline & PT visit & & 1 & 4 & 3 & 2 & 1 & 1 & & & & & & & & & & & $0.064-0.75$ & 0.19 & 0.5 \\
\hline
\end{tabular}

MEF middle ear fluid, nasopharyngeal (NP)/oropharyngeal (OP) samples, PT post-treatment 


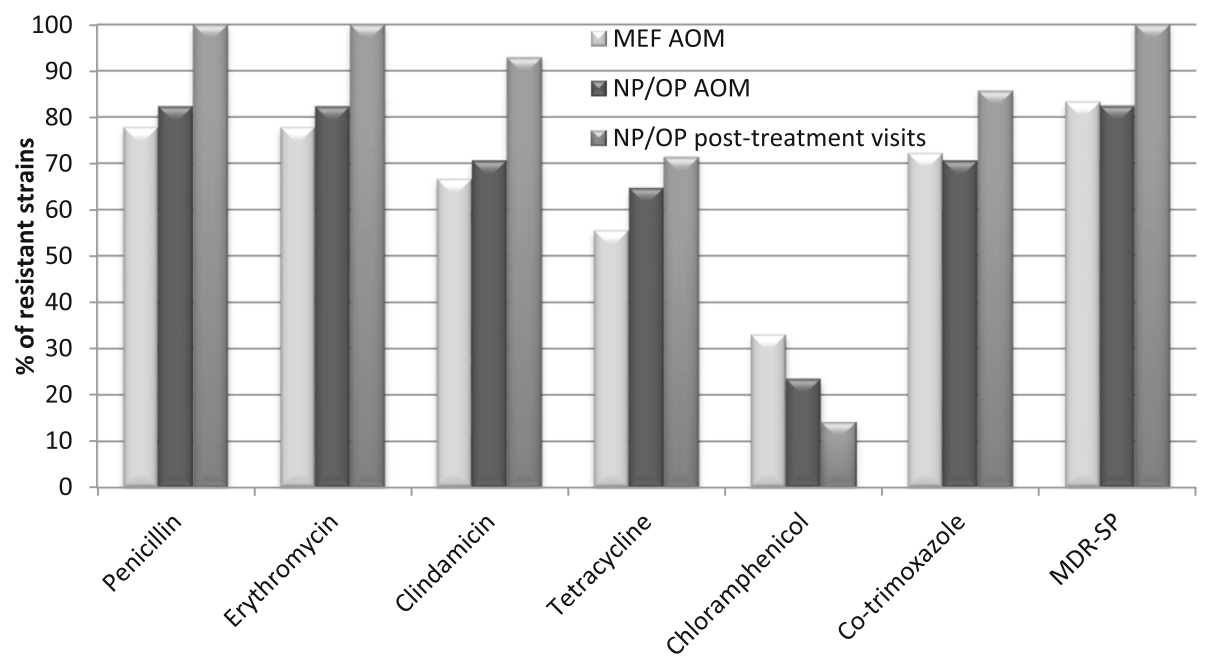

Fig. 3 Resistance to antibiotics for 55 pneumococcal isolates obtained during AOM and post-treatment visits. (AOM, acute otitis media; MEF, middle ear fluid; NP, nasopharyngeal; OP, oropharyngeal; MDR-SP, multidrug resistant S. pneumoniae)

both at the AOM $(p=0.015)$ and during post-treatment visits $(p=0.047)$. However, in one immunized patient $S$. pneumoniae isolate with serotype $6 \mathrm{~B}$ was detected in MEF sample. A number of studies have examined the impact of antipneumococcal immunization with the use of conjugate vaccines on the burden of AOM. The reduction of visits for AOM, antibiotic prescription and tympanosomy tube placements were noted in these studies [13,36]. However, in the Belgian study of children with the history of recurrent $\mathrm{AOM}$ immunized with PCV7 followed by a 23-valent polysaccharide booster, the authors reported no reduction in the number of AOM episodes and no changes in nasopharyngeal pneumococcal carriage during the 26-month follow-up period suggesting that the vaccine may not be useful when recurrent AOM is established [37].

After eight years since PCV7 introduction in United States it was reported that PCV7 serotypes virtually disappeared from MEF of vaccinated children with AOM [38]. High rate (73.7-80.1\%) of PCVs serotype coverage of isolates colonizing upper respiratory tract in pre-school healthy children as well as in children with recurrent upper respiratory tract infections from south-east Poland was observed [20, 39]. According to Skoczynska et al. study from Poland [40], in children aged less than 5 years, the PCV10, and PCV13 covered $54.8 \%$, and $68.8 \%$ of all IPD cases, and $76.3 \%$, and $86.3 \%$ of cases involving children under 5 years of age. Our data showed that the $S$. pneumoniae serotype coverage by the currently available PCVs among isolates colonizing nasopharynx as well as otopathogens from MEF cultures in children with AOM from Poland is still very high (84-92\%) and exceeded these reported in other European countries [35, 41]. A routine antipneumococcal vaccination has only been implemented in Poland in 2017 (https://gis.gov.pl/en/ homepage/). Two years before, the PCVs had been recommended for children under 5 years old however with reimbursement only for sselected risk groups. National Institute of Public Health of National Institute of Hygiene annual data revealed slightly increasing percentage (from 1.5 to $4 \%$ ) of vaccinated children aged 0-14 years in 2007-2014 period (http://wwwold.pzh.gov.pl/oldpage/epimeld/index_a.html).

In the present study, of the 10 clonal complexes recovered, all have previously been described in other countries, including 3 of the 43 worldwide spread resistant pneumococcal strains currently accepted by PMEN, Spain 9 V-3 (ST156), England 14-9 (ST9) and Spain 23F-1 (ST81). Spain23F-1 (ST81) and Spain9V-3 (ST156) have been present in Poland since the second half of the 1990s [2, 42]. A significant decrease in Spain23F-1 prevalence in some regions [43, 44] has been observed since the introduction of a seven-valent pneumococcal conjugate vaccine (PCV7) in 2001. Also in Poland the decrease of the Spain23F-1 clone occurrence from 19.1 to $6.8 \%$ among invasive and non-invasive PNSSP isolates was noticed [2, 42]. In our study, this clone constituted $10 \%$ of PNSSP isolates. Spain23F-1 is considered as a clone with low deposition of causing invasive diseases, and its adaptation to persistent colonization of the human nasopharynx may facilitate its intercontinental distribution [45]. The spreading of two related clones Spain9V-3 (ST156) and ST143, and their representatives seems to be responsible for the increase in resistance observed in 2002 in Poland [42]. Between 2002 and 2005, the Spain9V-3 complex spread in Poland from 22 to $47.5 \%$ of PNSSP isolates [2, 42]. In our study, CC1 (ST156/ST143/ST10340) 
isolates represented $30 \%$ of PNSSP isolates. Penicillin nonsusceptible variants of England14-9 (ST9) for the first time have been reported in Poland in 20042005 years [2] and in our study this clone with variants represented $20 \%$ of tested pneumococcal strains. Some of the previously detected clones [19] were found again, including ST87, ST135 and ST320 strains.

\section{Conclusions}

The high persistent prevalence of antibiotic-resistant $S$. pneumoniae strains in children with $\mathrm{AOM}$ after the completion of the therapy seems to confirm that the unsuccessful bacterial eradication may be regarded as a risk factor of infection recurrence. Presence of PCV serotypes as well as PMEN resistant clones in tested children are confirmation of their spreading caused by minimal uptake of PCVs in Polish population.

\section{Additional file}

Additional file 1: Analysis of the similarity among tested isolates and PMEN strains made by eBURSTV3 software. (DOCX $56 \mathrm{~kb}$ )

\begin{abstract}
Abbreviations
AOM: Acute Otitis Media; CC: Clonal Compex; Cl: Confidence Intervals; CRP: C-Reactive protein; DCC: Day care center; ENT: Ear-Nose-and-Throat; EUCAST: European Committee on Antimicrobial Susceptibility Testing; Hib: Haemophilus influenzae type b; MEF: Middle Ear Fluid; MLST: Multilocus Sequence Typing; N: New case AOM; NP: Nasopharyngeal; OP: Oropharyngeal; OR: Odds Ratio; PMEN: Pneumococcal Molecular Epidemiology Network; PNSSP: Penicillin Non-Susceptible S. pneumoniae; PSSP: Penicillin Susceptible S. pneumoniae; R: Recurrent AOM; RR: Relative risk; Sp: Streptococcus pneumoniae; ST: Sequence Type; TF: AOM treatment failure; WBC: White blood cells
\end{abstract}

\section{Acknowledgements}

The paper was developed using the equipment purchased within the agreement No. POPW.01.03.00-06-010/09-00 Operational Program Development of Eastern Poland 2007-2013, Priority Axis I, Modern Economy, Operations 1.3. Innovations Promotion.

\section{Availability of data and materials}

The datasets used and/or analyzed during the current study available from the corresponding author on reasonable request.

\section{Authors' contributions}

Performed the experiments: IKG, PZ, RS, EM. Analyzed the data: IKG, AM, PZ. Contributed to clinical management of patients: PZ, GN. Wrote the paper: IKG, AM. All authors were involved at study conception and design stage. All authors read and approved the final manuscript.

\section{Ethics approval and consent to participate}

The Ethical Committee of the Medical University of Lublin approved the study protocol (No. KE-0254/75/211). From all children's parents written informed consent was obtained.

\section{Consent for publication}

Not applicable.

\section{Competing interests}

The authors declare that they have no competing interests.

\section{Publisher's Note}

Springer Nature remains neutral with regard to jurisdictional claims in published maps and institutional affiliations.

\section{Author details}

${ }^{1}$ Department of Pharmaceutical Microbiology with Laboratory for Microbiological Diagnostics, Medical University of Lublin, Chodzki 1 Street, 20-093 Lublin, Poland. ²Department of Pediatric Otolaryngology, Phoniatrics and Audiology, Medical University of Lublin, Lublin, Poland. ${ }^{3}$ Department of Medical Microbiology, Medical University of Lublin, Lublin, Poland.

Received: 23 October 2017 Accepted: 19 September 2018

Published online: 25 September 2018

\section{References}

1. Bogaert D, de Groot R, Hermans PWM. Streptococcus pneumoniae colonization: the key to pneumococcal disease. The Lancet Infect Dis. 2004;4:144-54. https://doi.org/10.1016/S1473-3099(04)00938-7.

2. Sadowy E, Kuch A, Gniadkowski M, Hryniewicz W. Expansion and evolution of the Streptococcus pneumoniae Spain9V-ST156 clonal complex in Poland. Antimicrob Agents Chemother. 2010;54:1720-7. https://doi.org/10.1128/ AAC.01340-09.

3. Siira L, Javala J, Tissari P, Vaara M, Kaijalainen T, Virolainen A. Clonality behind the increase of multidrug-resistance among non-invasive pneumococci in southern Finland. Eur J Clin Microbiol Infect Dis. 2012;31: 867-71. https://doi.org/10.1007/s10096-011-1386-8.

4. Geng Q, Zhang T, Ding Y, Tao Y, Lin Y, Wang Y, et al. Molecular characterization and antimicrobial susceptibility of Streptococcus pneumoniae isolated from children hospitalized with respiratory infections in Suzhou, China. PlosOne. 2014;9:e93752. https://doi.org/10.1371/journal. pone.0093752.

5. McGee L, McDougal L, Zhou J, et al. Nomenclature of major antimicrobialresistant clones of Streptococcus pneumoniae defined by the pneumococcal molecular epidemiology network. J Clin Microbiol. 2001;9:2565-71. https://doi.org/10.1128/JCM.39.7.2565-2571.2001

6. O'Brien KL, Wolfson LJ, Watt JP, et al. Burden of disease caused by Streptococcus pneumoniae in children younger than 5 years: global estimates. Lancet. 2009;374:893-902. https://doi.org/10.1016/S0140-6736(09)61204-6.

7. Isaacman DJ, Mclntosh ED, Reinert RR. Burden of invasive pneumococcal disease and serotype distribution among Streptococcus pneumoniae isolates in young children in Europe: impact of the 7-valent pneumococcal conjugate vaccine and considerations for the future conjugate vaccines. Int J Infect Dis. 2010;14:e197-209. https://doi.org/10.1016/j.ijid.2009.05.010.

8. Teele DW, Klein JO, Rosner B. Epidemiology of otitis media during the first seven years of life in children in greater Boston: a prospective, cohort study. J Infect Dis. 1989;160:83-94.

9. Vergison A. Microbiology of otitis media: a moving target. Vaccine. 2008; 26(Suppl 7):G5-10. https://doi.org/10.1016/j.vaccine.2008.11.006.

10. Korona-Glowniak I, Mazur E, Zychowski P, Niedzielska G, Koziol-Montewka M MalmA. Bacterial etiology of recalcitrant acute otitis media in sixty two children - high risk of pathogen colonization after treatment. Clin. Otolaryngology. 2018;43:665-9. https://doi.org/10.1111/coa.12986.

11. Vergison A, Dagan R, Arguedas A, et al. Otitis media and its consequences: beyond the earache. Lancet Infect Dis. 2010;10:195-203. https://doi.org/10. 1016/S1473-3099(10)70012-8.

12. Pichichero ME, Pichichero CL. Persistent acute otitis media: II. Antimicrobial treatment Pediatr Infect Dis J. 1995;14:183-8.

13. Dupont $D$, Mahjoub-Messai F, François $M$, et al. Evolving microbiology of complicated acute otitis media before and after introduction of the pneumococcal conjugate vaccine in France. Diagn Microbiol Infect Dis. 2010;68:89-92. https://doi.org/10.1016/j.diagmicrobio.2010.04.012.

14. Leibowitz E. Complicated otitis media and its implications. Vaccine. 2008;26S:G16-9. https://doi.org/10.1016/j.vaccine.2008.11.008.

15. Libson S, Dagan R, Greenberg D, et al. Nasopharyngeal carriage of Streptococcus pneumoniae at the completion of successful antibiotic treatment of acute otitis media predisposes to early clinical recurrence. J Infect Dis. 2005;191:1869-75. https://doi.org/10.1086/429918.

16. Bosch AA, Biesbroek G, Trzcinski K, Sanders EA, Bogaert D. Viral and bacterial interactions in the upper respiratory tract. PLoS Pathog. 2013;9:e1003057. https://doi.org/10.1371/journal.ppat.1003057. 
17. Peters BM, Jabra-Rizk MA, O'May GA, Costerton JW, Shirtliff ME. Polymicrobial interactions: impact on pathogenesis and human disease Clin Microbiol Rev. 2012;25:193-213. https://doi.org/10.1128/CMR.00013-11.

18. Bluestone CD. Definitions, terminology, and classification. In: Bluestone CD, Klein JO, Decker BC, editors. Otitis media in infants and children. 4th ed. Canada: Hamilton; 2007.

19. Korona-Glowniak I, Maj M, Siwiec R, Niedzielski A, Malm A. Molecular epidemiology of Streptococcus pneumoniae isolates from children with recurrent upper respiratory tract infections. PLoS One. 2016;11:e0158909. https://doi.org/10.1371/journal.pone.0158909.

20. Niedzielski A, Korona-Glowniak I, Malm A. High prevalence of Streptococcus pneumoniae in adenoids and nasopharynx in preschool children with recurrent upper respiratory tract infections in Poland--distribution of serotypes and drug resistance patterns. Med Sci Monit. 2013;19:54-60. https://doi.org/10.12659/MSM.883742.

21. Leibowitz E. The challenge of recalcitrant acute otitis media: pathogens, resistance, and treatment strategy. Pediatr Infect Dis J. 2007;26(Suppl. 10): S8-11. https://doi.org/10.1097//NF.0b013e318154b24a.

22. Pichichero ME, Casey JR. Emergence of a multiresistant serotype 19A pneumococcal strain not included in the 7-valent conjugate vaccine as an otopathogen in children. JAMA. 2007;298:1772-8. https://doi.org/10.1001/ jama.298.15.1772.

23. Eldan M, Leibovitz E, Piglansky L, et al. Predictive value of pneumococcal nasopharyngeal cultures for the assessment of nonresponsive acute otitis media in children. Pediatr Infect Dis J. 2000;19:298-303.

24. Stol K, Verhaegh SJ, Graamans K, Engel JA, Sturm PD, Melchers WJ, Meis JF, Warris A, Hays JP, Hermans PW. Microbial profiling does not differentiate between childhood recurrent acute otitis media and chronic otitis media with effusion. Int J Pediatr Otorhinolaryngol. 2013;77:488-93. https://doi.org/ 10.1016/j.jporl.2012.12.016.

25. Hotomi M, Yamanaka N, Billal DS, Sakai A, Yamauchi K, Suzumoto M, Takei S, Yasui N, Moriyama S, Kuki K. Genotyping of Streptococcus pneumoniae and Haemophilus influenzae isolated from paired middle ear fluid and nasopharynx by pulsed-field gel electrophoresis. ORL J Otorhinolaryngol Relat Spec. 2004;66:233-40. https://doi.org/10.1159/000081119.

26. Tonnaer EL, Rijkers GT, Meis JF, Klaassen CH, Bogaert D, Hermans PW, Curfs $\mathrm{JH}$. Genetic relatedness between pneumococcal populations originating from the nasopharynx, adenoid, and tympanic cavity of children with otitis media. J Clin Microbiol. 2005;43:3140-4. https://doi.org/10.1128/JCM.43.7. 3140-3144.2005.

27. Rodriguez WJ, Schwartz RH. Streptococcus pneumoniae causes otitis media with higher fever and more redness of tympanic membranes than Haemophilus influenzae or Moraxella catarrhalis. Pediatr Infect Dis J. 1999;18:942-4.

28. Palmu AA, Herva E, Savolainen H, Karma P, Mäkelä PH, Kilpi TM. Association of clinical signs and symptoms with bacterial findings in acute otitis media. Clin Infect Dis. 2004;38:234-42. https://doi.org/10.1086/380642.

29. McCormick DP, Lim-Melia E, Saeed K, Baldwin CD, Chonmaitree T. Otitis media: can clinical findings predict bacterial or viral etiology? Pediatr Infect Dis J. 2000;19:256-8.

30. Barkai G, Leibovitz E, Givon-Lavi N, Dagan R. Potential contribution by nontypable Haemophilus influenzae in protracted and recurrent acute otitis media. Pediatr Infect Dis J. 2009;28:466-71. https://doi.org/10.1097/INF. Ob013e3181950c74.

31. Mayanskiy N, Alyabieva N, Ponomarenko O, et al. Bacterial etiology of acute otitis media and characterization of pneumococcal serotypes and genotypes among children in Moscow, Russia. Pediatr Infect Dis J. 2015;34: 255-60. https://doi.org/10.1097/INF.0000000000000554.

32. Zielnik-Jurkiewicz B, Bielicka A. Antibiotic resistance of Streptococcus pneumoniae in children with acute otitis media treatment failure. Int J Pediatr Otorhinolaryngol. 2015;79:2129-33. https://doi.org/10.1016/j.jporl. 2015.09.030

33. Ruohola A, Meurman O, Nikkari S, Skottman T, Salmi A, Waris M, Osterback R, Eerola E, Allander T, Niesters H, Heikkinen T, Ruuskanen O. Microbiology of acute otitis media in children with tympanostomy tubes: prevalences of bacteria and viruses. Clin Infect Dis. 2006;43:1417-22. https://doi.org/10. 1086/509332.

34. Nourizadeh N, Ghazvini K, Gharavi V, et al. Evaluation of nasopharyngeal microbial flora and antibiogram and its relation to otitis media with effusion. Eur Arch Otorhinolaryngol. 2016;273:859-63. https://doi.org/10. 1007/s00405-015-3637-2
35. Pumarola F, Marès J, Losada I, Minguella I, Moraga F, Tarragó D, Aguilera U, Casanovas JM, Gadea G, Trías E, Cenoz S, Sistiaga A, García-Corbeira P, Pirçon JY, Marano C, Hausdorff WP. Microbiology of bacteria causing recurrent acute otitis media (AOM) and $\mathrm{AOM}$ treatment failure in young children in Spain: shifting pathogens in the post-pneumococcal conjugate vaccination era. Int J Pediatr Otorhinolaryngol. 2013;77:1231-6. https://doi. org/10.1016/j.ijporl.2013.04.002.

36. Zhou F, Shefer A, Kong Y, et al. Trends in acute otitis media-related health care utilization by privately insured young children in the United States, 1997-2004. Pediatrics. 2008;121:253-60. https://doi.org/10.1542/peds.2007-0619.

37. Veenhoven $\mathrm{R}$, Bogaert $\mathrm{D}$, Uiterwaal $\mathrm{C}$, et al. Effect of conjugate pneumococcal vaccine followed by polysaccharide pneumococcal vaccine on recurrent acute otitis media: a randomised study. Lancet. 2003;361:2189-95. https://doi.org/10. 1016/S0140-6736(03)13772-5.

38. Casey JR, Adlowitz DG, Pichichero ME. New patterns in the otopathogens causing acute otitis media six to eight years after introduction of pneumococcal conjugate vaccine. Pediatr Infect Dis J. 2010;29:304-9. https://doi.org/10.1097/INF.0b013e3181c1bc48.

39. Korona-Glowniak I, Niedzielski A, Malm A, Niedzielska G. Serotypes and antibiotic resistance of Streptococcus pneumoniae from adenoids in preschool children with recurrent upper respiratory tract infections. Pol J Microbiol. 2013:62:385-90.

40. Skoczyńska A, Kuch A, Sadowy E, et al. Recent trends in epidemiology of invasive pneumococcal disease in Poland. Eur J Clin Microbiol Infect Dis. 2015;34:779-87. https://doi.org/10.1007/s10096-014-2283-8.

41. McIntosch ED, Fritzell B, Fletcher MA. Burden of paediatric invasive pneumococcal disease in Europe, 2005. Epidemiol Infect. 2007;135:644-56. https://doi.org/10.1017/S0950268806007199.

42. Sadowy E, Izdebski R, Skoczynska A, Grzesiowski P, Gniadkowski M, Hryniewicz W. Phenotypic and molecular analysis of penicillinnonsusceptible Streptococcus pneumoniae isolates in Poland. Antimicrob Agents Chemother. 2007:51:40-7. https://doi.org/10.1128/AAC.01072-06.

43. Calatayud L, Ardanuy C, Cercenado E, Fenoll AD, Bouza E, Pallarés R, Martín $\mathrm{R}$, Liñares J. Serotypes, clones, and mechanisms of resistance of erythromycin-resistant Streptococcus pneumoniae collected in Spain. Antimicrob Agents Chemother. 2007;51:3240-6. https://doi.org/10.1128/ AAC.00157-07.

44. Jacobs MR, Good CE, Beall B, Bajaksouzian S, Windau AR, Whitney CG. Changes in serotypes and antimicrobial susceptibility of invasive Streptococcus pneumoniae strains in Cleveland: a quarter century of experience. J Clin Microbiol. 2008;46:982-90. https://doi.org/10.1128/JCM.02321-07.

45. Croucher NJ, Walker D, Romero P, Lennard N, Paterson GK, Bason NC, Mitchell AM, Quail MA, Andrew PW, Parkhill J, Bentley SD, Mitchell TJ. Role of conjugative elements in the evolution of the multidrug-resistant pandemic clone Streptococcus pneumoniae Spain23F ST81. J Bacterial. 2009;191:1480-9. https://doi.org/10.1128/JB.01343-08.

Ready to submit your research? Choose BMC and benefit from:

- fast, convenient online submission

- thorough peer review by experienced researchers in your field

- rapid publication on acceptance

- support for research data, including large and complex data types

- gold Open Access which fosters wider collaboration and increased citations

- maximum visibility for your research: over $100 \mathrm{M}$ website views per year

At BMC, research is always in progress.

Learn more biomedcentral.com/submissions 